\title{
Research on Construction Schedule Optimization of Assembly Building Based on NSGA-II
}

\author{
Ru Wang ${ }^{1,2}$, Jing $\operatorname{Lian}^{1 *}$ \\ ${ }^{1}$ College of Civil Engineering, Xi'an University of Architecture \& Technology, Xi'an, shaanxi, 710000, China \\ ${ }^{2}$ Collaborative Innovation Center for Assembled Buildings in Western China (XAUAT), Xi'an, shaanxi, 710000, China
}

\begin{abstract}
Considering multiple possible scenarios in the process of project construction, the prefabricated project scheduling problem is studied in combination with the theory of multi-mode resource-constrained project scheduling problem. Multi-objective multi-mode resource-constrained project scheduling model with time/robustness trad-offs was constructed. Next, the adjusted non-dominated genetic algorithm (NSGA-II) was designed to solve the model. Finally, the proposed model and algorithm were implicated to a real project, in order that research achievement can guide managers to make decisions and invest resources scientifically and reasonably.
\end{abstract}

\section{Introduction}

Because of the high degree of mechanization, prefabricated buildings have become the development trend of the current construction industry. The scheduling problem of prefabricated engineering has become a hot topic. Jeonghoon Lee[1] proposed a modular construction project scheduling model based on genetic algorithm, aiming at the problem that the current project scheduling technology cannot deal with the whole project process at the same time. Taghaddos[2] proposed a simulation-based auction protocol (SBAP) to solve the problem of how to allocate resources efficiently in prefabricated projects under various constraints. Clyde Li C Z[3] developed a hybrid dynamic model using hybrid system dynamics and discrete event simulation to evaluate the impact of schedule risk on the schedule of prefabricated buildings.Jiang $\mathrm{H}$ Y [4] proposed an improved PERT model based on critical chain method with the objective of minimum duration under the constraints of logical relationship and resources. Chen $\mathrm{W}[5,6]$ constructed a multi-space collaborative scheduling model for the whole process of production, transportation and site installation of prefabricated building components, but he did not give a solution to the model. Although Wu H[7] and Wang X[8] studied the problem from the perspective of robustness of scheduling, the method of taking robustness as a constraint condition still needs to be improved. According to the existing literature, the research results of prefabricated project scheduling are not rich enough, so it is still necessary to further study, so as to provide scientific decision-making basis for project managers to make a schedule.

In this paper, we study the scheduling problem of prefabricated buildings from the perspective of time/robustness trade-off optimization. Firstly, the biobjective multi-mode resource constraint project scheduling model was constructed. Next, the adjusted NSGA-II was designed to solve the model. Finally, a real construction project was analyzed as an example.

\section{Problem statement and model establishment}

\subsection{Notations and definitions}

There are many uncertainties in construction projects due to severe changes in the climate, resource consumption fluctuation or site conditions. How to make reasonable schedule with short duration and strong resistance to uncertainties is one of the most significant tasks for project managers. So it is the best way to to study problems of prefabricated project schedule based on the theory multi-mode resource constraint project scheduling problem (MRCPSP). The bi-objective MRCPSP for duration/ robustness trade-off is described as follows. A total of $\mathrm{K}$ renewable resources are required for the smooth implementation, and the maximum supply of $k_{\text {th }}$ resource is expressed as $R_{k}$. The activity-on-node $\mathrm{G}=(\mathrm{V}, \mathrm{N})$ is adopted to represent project network. The set of nodes $\mathrm{V}$ denotes processes, $\mathrm{V}=\{1, \ldots, \mathrm{J}\}$.Each process $\mathrm{J}_{\mathrm{j}}$ has $\mathrm{M}$ optional execution modes, characterized by a combination of duration/resources usage $\left(\mathrm{d}_{\mathrm{jm}}, \mathrm{r}^{\mathrm{k}} \mathrm{jm}\right)$, where $\mathrm{r}^{\mathrm{k}}{ }_{\mathrm{jm}}$ is the amount of $\mathrm{k}_{\mathrm{th}}$ resources consumed when process $\mathrm{j}$ is executed in $\mathrm{m}$ mode; $\mathrm{d}_{\mathrm{jm}}$ is its duration required. The set of directed arcs $\mathrm{N}$ represents the finish - start, zero-lag precedence relations. The nodes are topologically numbered from the single start node 1 to the single terminal node $\mathrm{J}, \mathrm{J}=|\mathrm{V}|$, where nodes 1 and $\mathrm{J}$ are dummy processes without consuming time and

\footnotetext{
*Corresponding author's e-mail: lianjing0325@163.com
} 
resources. $P(j)$ is used to represent the set of immediate predecessors of process $\mathrm{j}$, and $\mathrm{S}(\mathrm{j})$ represents its set of successors.

\subsection{Establishment of BOMRCPSP model}

According to the previous description of the problem and the symbolic description, the model studied in this paper can be expressed as follows:

objective function: $\min T=f_{J}$

$$
\begin{gathered}
R M=\sum\left(|S(j)| * \lambda_{j m} * \sum_{n=1}^{f f_{j}^{r c}} e^{-n}\right) \\
\text { subject to: } \sum_{m=1}^{M_{j}} x_{j m}=1, j \in V \\
f_{j}-\sum_{m} x_{j m} * d_{j m} \geq f_{i},(i, j) \in N \\
\sum_{j \in A_{t}} \sum_{m} x_{j m} * r_{j m}^{k} \leq R_{k} \\
x_{j m}=\{0,1\}, j \in V
\end{gathered}
$$

In the above model, $f_{j}$ represents the finish time of process $\mathrm{j}$, and equation (1) is project duration. Equation (2) expresses robustness value, where $\lambda_{j m}=\sum_{k} r_{j m}^{k} / R_{k}$ indicating the contribution coefficient of each activity to the robustness of the schedule, $|\mathrm{S}(\mathrm{j})|$ represents the number of successors and $\mathrm{ff}^{\mathrm{rc}}{ }_{\mathrm{jm}}$ represents free slack of activity $\mathrm{j}$ under resource constraints; the equation (3) indicates that each activity can only be performed by one mode; the equation (4) represents the relationship between activities. The decision variables $\mathrm{s}_{\mathrm{j}}$ is calculated by $\mathrm{s}_{\mathrm{j}}=\mathrm{f}_{\mathrm{j}}-\mathrm{d}_{\mathrm{jm}}$. Constraint (5) indicates that the demand of

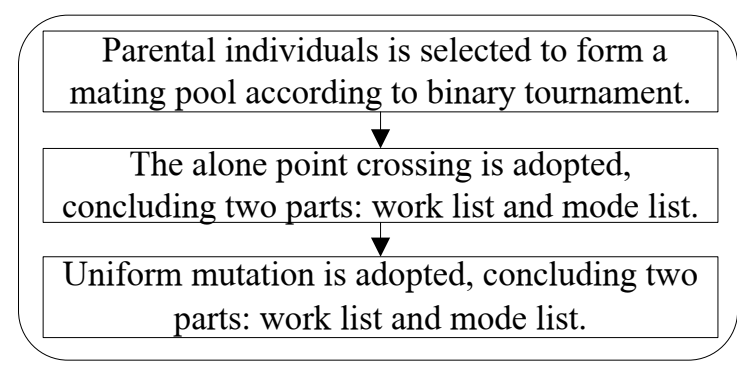

A new population is formed by merging parent population with progeny population.

Calculating non-dominant sort and crowding distance for the combined population.

The next generation population Pgen is formed based on the elitist strategy. $\mathrm{k}_{\text {th }}$ resource for all activities executed shall not exceed its allowance. $A_{t}$ represents the set of all processes being performed at the moment. The equation (6) indicates the range of the decision variable $\mathrm{x}_{\mathrm{jm}}$.

\section{Adjusted NSGA-II to solve BOMRCPSP model}

\subsection{Coding and Decoding}

This paper adopts the method of real number coding [10], as shown in Figure 1. The length of the each chromosome is $2 * \mathrm{~J}$, which is denoted as $\mathrm{I}=(\lambda, \mu)$, where the former $J$ genes $\lambda$ are sequences that satisfy the order constraint between processes; the remaining $\mu$ are executive mode genes corresponding to the former one by one. We adopted serial schedule generation scheme(SSGS) to compute the start time of every activity,which is the earliest finish times of its all predecessors under resources constraint. Thus, the makespan is obtained obviously. After that robustness values are calculated through equations (2).

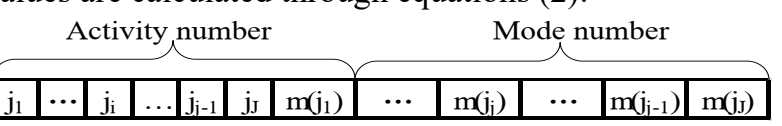

Figure1 Coding of chromosome.

\section{2. the Adjusted NSGA-II.}

The process of adjusted NSGA-II for solving project scheduling problem of prefabricated buildings is shown in Figure2.

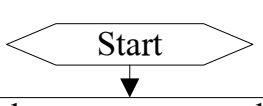

Initializing the parameters and generating initial population

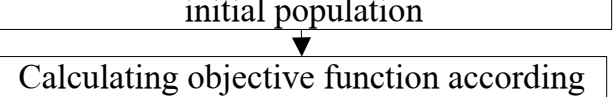

to serial scheduling Generation scheme
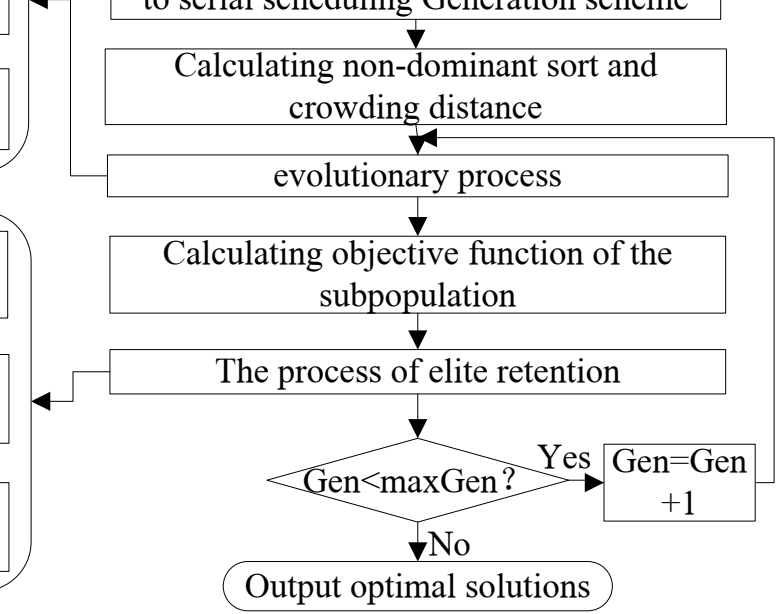

Figure2 the Process of Adjusted NSGA-II. 
3.2.1. Alone Point Crossover. The $\mathrm{I}^{\mathrm{F}}$ and $\mathrm{I}^{\mathrm{M}}$ are two parent chromosomes, and the two progeny chromosomes are recorded as $\mathrm{I}^{\mathrm{D}}$ and $\mathrm{I}^{\mathrm{S}}$. When the activity vector is crossed, firstly, the random number $\mathrm{q}_{1}\left(1<\mathrm{q}_{1}<\mathrm{J}\right)$ was generated as the intersection point, and then activity vector of $\mathrm{I}^{\mathrm{D}}$ was generated as follows: the genes from 1 to $q_{1}$ inherited from $I^{M}$, while the genes from $q_{1}+1$ to $J$ inherited the $\mathrm{I}^{\mathrm{F}}$, which are different from $\mathrm{q}$ genes of $\mathrm{I}^{\mathrm{D}}$. When the mode vector is crossed, a random number $r_{1}\left(1<r_{1}<J\right)$ was also generated as a cross point. The first $r_{1}$ genes in the $\mathrm{x}^{\mathrm{D}}$ mode vector followed genes corresponding to $\mathrm{x}^{\mathrm{M}}$, and the genes from $\mathrm{r}_{1}+1$ to $\mathrm{J}$ inherited from $x^{\mathrm{F}}$. Identically, the $\mathrm{x}^{\mathrm{S}}$ mode vector inheritance was opposite to the $\mathrm{I}^{\mathrm{D}}$.

3.2.2. Uniform mutation. Each gene has a probability $\mathrm{P}_{\mathrm{m}}$ to be selected to mutate. The mutation process of activity vector can be seen in Zhang[9].For the mode vector, one of its genes is randomly selected and its value is randomly changed to a different mode. The mode vector does not change when the activity vector mutates.

\section{Case study}

\subsection{Project Introduction}

In order to illustrate the versatility and applicability of the proposed model and algorithm, a real project was analyzed as an example. The project was constructed in three areas. The construction in one of these areas was taken as an object of the research. There are two optional modes for each process (One mode was regarded as two identical modes for the convenience of calculation). The supply of resources for this project are as follows:(1) managers:8person/day;(2)lifting equipment:36 machineteam/day;(3)laborers:18 person/day. Table 1 gives relative basic data of each activity. The activity-on-node network of project is shown in figure 3 .

Table 1. List of Project Information

\begin{tabular}{|c|c|c|c|c|c|c|}
\hline \multirow[b]{2}{*}{ Process } & \multirow{2}{*}{$\begin{array}{l}\text { Process } \\
\text { number }\end{array}$} & \multirow{2}{*}{$\begin{array}{l}\text { Mode } \\
\text { number }\end{array}$} & \multirow[b]{2}{*}{$\begin{array}{l}\text { Duration } \\
\text { /day }\end{array}$} & \multicolumn{3}{|c|}{ amount of Resources demand } \\
\hline & & & & Managers & $\begin{array}{l}\text { Lifting } \\
\text { Equipment }\end{array}$ & Laborers \\
\hline Starting & 1 & 1 & 0 & 0 & 0 & 0 \\
\hline Construction preparation & 2 & 1 & 1 & 6 & 0 & 0 \\
\hline Plant bolt of $A \backslash B$ & $3 \backslash 14$ & 1 & 1 & 2 & 0 & 6 \\
\hline \multirow{2}{*}{ Column lifting of $\mathrm{A} \backslash \mathrm{B}$} & \multirow{2}{*}{$4 \backslash 15$} & 1 & 2 & 3 & 36 & 9 \\
\hline & & 2 & 3 & 2 & 24 & 6 \\
\hline \multirow{2}{*}{$\begin{array}{l}\text { Mounting bracing of } A \backslash B \\
\text { Grouting of } A \backslash B\end{array}$} & $5 \backslash 16$ & 1 & 1 & 3 & 0 & 4 \\
\hline & $6 \backslash 17$ & 1 & 1 & 2 & 0 & 4 \\
\hline \multirow{2}{*}{ Single beam lifting of $\mathrm{A} \backslash \mathrm{B}$} & \multirow{2}{*}{$7 \backslash 18$} & 1 & 1 & 2 & 32 & 8 \\
\hline & & 2 & 2 & 2 & 24 & 6 \\
\hline Beam protection of $A \backslash B$ & $8 \backslash 19$ & 1 & 1 & 2 & 0 & 4 \\
\hline \multirow{2}{*}{ Lattice beam lifting of $A \backslash B$} & \multirow[b]{2}{*}{$9 \backslash 20$} & 1 & 1 & 4 & 36 & 12 \\
\hline & & 2 & 2 & 3 & 24 & 8 \\
\hline \multirow{2}{*}{$\begin{array}{l}\text { Sleeve, bolt connecting of } \\
\text { A/B connection }\end{array}$} & \multirow{2}{*}{$10 \backslash 21$} & 1 & 1 & 4 & 0 & 10 \\
\hline & & 2 & 2 & 3 & 0 & 8 \\
\hline Gluing, grouting of $\mathrm{A} / \mathrm{B}$ & $11 \backslash 22$ & 1 & 1 & 3 & 0 & 6 \\
\hline \multirow{2}{*}{ Plywood hoisting of A/B } & \multirow{2}{*}{$12 \backslash 23$} & 1 & 1 & 3 & 36 & 12 \\
\hline & & 2 & 2 & 2 & 24 & 8 \\
\hline Concrete pouring of A/B & $13 \backslash 24$ & 1 & 1 & 4 & 0 & 4 \\
\hline The end & 25 & 1 & 0 & 0 & 0 & 0 \\
\hline
\end{tabular}

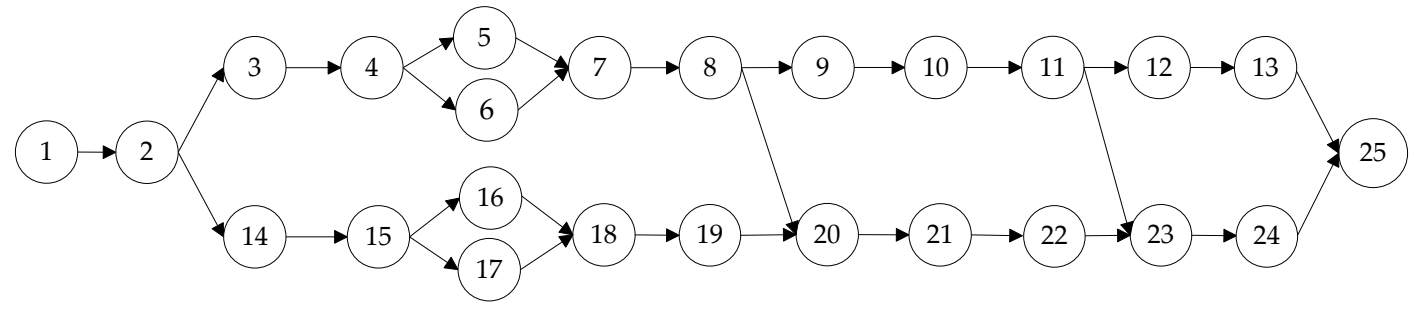

Figure 3. Activity-On-Node of the Project 


\subsection{Results}

We implemented the proposed algorithm in MATLABR2014b on computers (Inter(R) Core (TM) i53230M,8GBRAM). The parameters of NSGA -II are set as follows: popsize $=120, \quad$ maxgen $=100, \quad \mathrm{Pc}=0.85$, $\mathrm{Pm}=0.10$. Pareto frontier is shown on figure 4 . It can be seen that Pareto solutions present a non-linear increasing distribution in the objective space, indicating that the longer the construction period, the stronger the robustness. Table 2 shows two schedules with minimum or maximal construction duration. Managers can choose schedule from obtained pareto solutions meeting actual requirements .

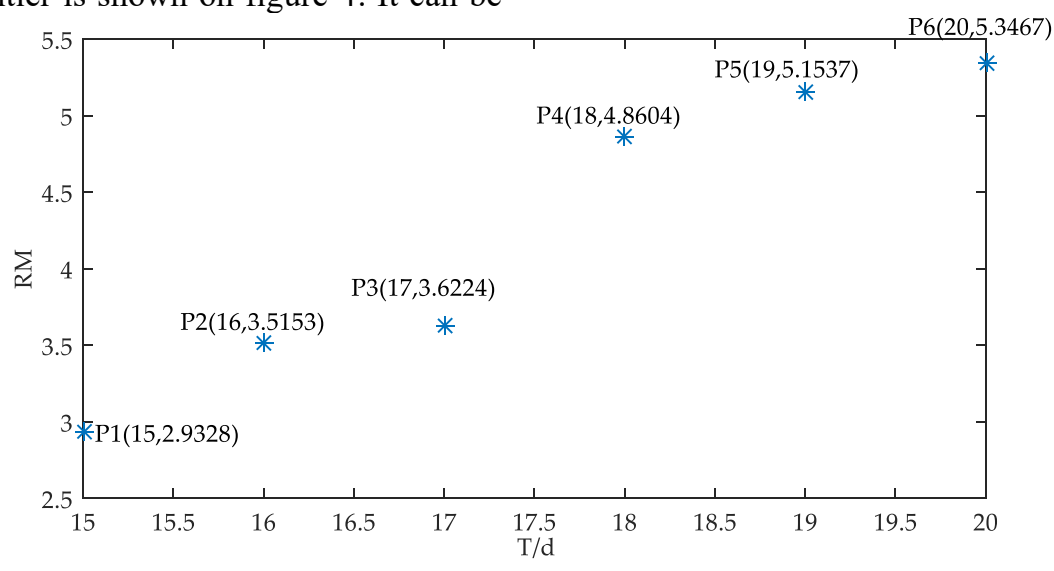

Figure 4 Optimized Duration-Robustness Pareto Frontier

Table 2. Schedules with minimum or maximal make-span

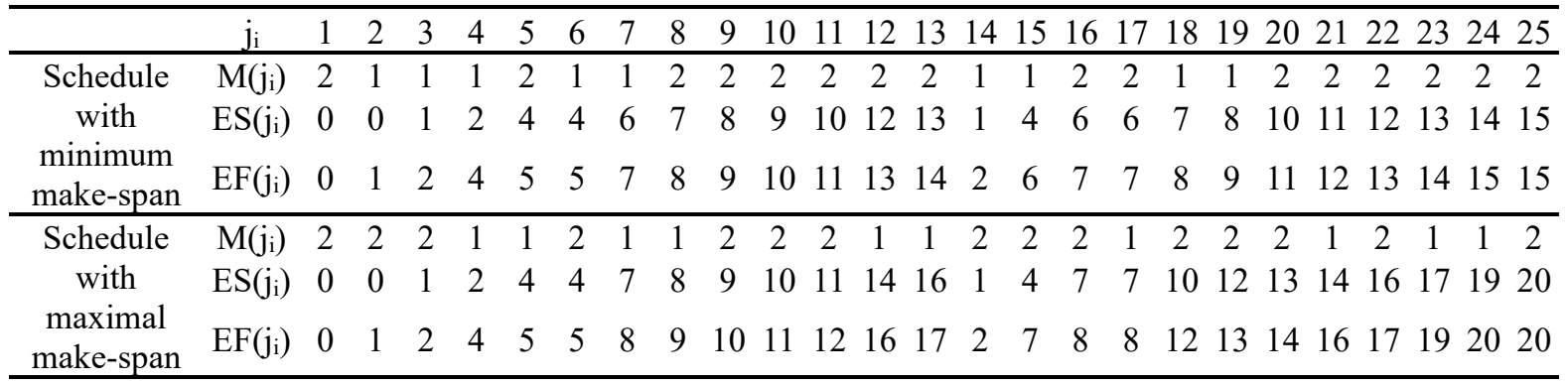

\section{Conclusion}

This paper studies the schedule problem of fabricated projects in uncertain environments. Firstly, a bi-objective multi-mode resource constraint project scheduling model (BOMRCPSP) with time/robustness trad-offs was established. Based on its feature multi-objective trade-off, an adjusted non-dominated genetic algorithm (NSGA-II) with elite retention strategy was designed, where crossover and mutation strategies were adjusted to satisfy constraints of the resources and priority relationships. Finally, the proposed BOMRCPSP model and adjusted NSGA-II were applied on a real construct project. The results show that the proposed method can solve the problem efficiently, and provide more scientific guidance for project managers.

\section{References}

1. Lee, J., \& Hyun, H. (2018). Multiple Modular Building Construction Project Scheduling Using Genetic Algorithms. Journal of Construction Engineering and Management, 145(1), 04018116.
2. Taghaddos, H., Hermann, U., AbouRizk, S., \& Mohamed, Y. (2014). Simulation-based multiagent approach for scheduling modular construction. Journal of Computing in Civil Engineering, 28(2), 263-274.

3. Li, C. Z., Hong, J., Fan, C., Xu, X., \& Shen, G. Q. (2018). Schedule delay analysis of prefabricated housing production: A hybrid dynamic approach. Journal of cleaner production, 195, 1533-1545.

4. Jiang, H, Y., Xie, X.H., \& Peng, Y. (2018). Improved PERT Model Based on Criticle Chain and Its Application of Assembly Building. Industrial Engineering and Management 23(5), 82-87.

5. Chen W, Qin H, L, \& Tong M, D. (2017). Resource scheduling of fabricated building engineering under multi-dimensional work space. Journal of Civil Engineering, 50(3), 115-122.

6. Chen W, Yu, Q.Y., Zhou M.et.al. (2018). Research on Schedule Buffers and Robustness of Prefabricated Building. Construction Econmy, 39(02), 33-39.

7. Wu, H. (2016). Research on the scheduling of the uncertain prefabricated housing project for the 
uncertain period (Master's thesis, Xi'an University of Architecture and Technology).

8. Wang, X. (2019). Study on Prefabricated Building Resource Scheduling Based on Improved Dolphin Swarm Algorithm (Master's thesis, Hebei University of Engineering).

9. Zhang, J. W., Zhou, S., \& Qiao, C. Z. (2018). A biobjective robust resource-constrained project scheduling problem with utility functions of activity floats. Journal of Systems \& Management, 27(2), 299-308. 